\title{
Evaluación de caracteres lineales de terneras Holstein F1 mediante el programa de cruzamiento ganadero Select Mating Service (SMS)
}

\author{
DOI: https://doi.org/10.33262/ap.v3i3.2.120
}

\begin{abstract}
(c) (1) (2)
Cy

Evaluation of linear traits of Holstein fl calves through the Select Mating Service (SMS) livestock crossing program
\end{abstract}

Diego Rodríguez Paspuel. ${ }^{1}$, Fabian Almeida López. ${ }^{2} \&$ Paula Toalombo Vargas. ${ }^{3}$

\begin{abstract}
The evaluation of the calves obtained through the Select Mating Service (SMS) crossbreeding program was carried out at the Tunshi Experimental Station, different improved phenotypic characters were evaluated through the application of the SMS program, genealogical records, in twelve calves of the mixed Holstein breed. The different variables evaluated were measured in absolute values, on a scale from 1 to 9 . The data were analyzed using excel software and descriptive results were obtained; The results showed a mean score for the angularity variable of 5.42 points; fortress 5.25 ; body depth 5.33 points; rump width 5.17 points; croup angle 5.58 points; rear view legs 6.17; legs side view 6.25 points; foot angle 5.25 points; height 5.50 points. Therefore, it can be said that there is an improvement in the linear characteristics of the calves evaluated, especially in legs that have a low heritability, which allows a longer permanence in the

\footnotetext{
1 Autor de la Investigación. Egresado de la Carrera de Ingeniería Zootécnica. FCP. ESPOCH, diegomau11@icloud.com, https://orcid.org/0000-0001-6086-70

2 Director del trabajo de Titulación. Docente FCP. ESPOCH, fabian.almeida@espoch.edu.ec, https://orcid.org/0000-0002-8728-3810

3 Miembro del Trabajo de Titulación. Docente FCP. ESPOCH, ptoalombo@espoch.edu.ec, https://orcid.org/0000-0002-7241-6852
} 
herd with an acceptable productive life and reducing the percentage of discards at age early.

Keywords: linear traits, body depth, heritability, Holstein.

\section{Resumen}

Se realizó la valoración de las terneras obtenidas mediante el programa de cruzamiento Select Mating Service (SMS), en la Estación Experimental Tunshi, se evaluaron diferentes caracteres fenotípicos mejorados mediante la aplicación del programa SMS, registros genealógicos, en doce terneras de la raza Holstein mestiza. Las diferentes variables evaluadas se midieron en valores absolutos, en una escala del 1 al 9. Los datos se analizaron mediante el software Excel y se obtuvieron resultados descriptivos; los resultados mostraron una puntuación media de la variable angulosidad de 5.42 puntos; fortaleza 5.25; profundidad corporal 5.33 puntos; anchura de grupa 5.17 puntos; ángulo de grupa 5.58 puntos; patas vista posterior 6.17; patas vista lateral 6.25 puntos; ángulo podal 5.25 puntos; estatura 5.50 puntos. Por lo que se puede decir que existe una mejora de las características lineales de las terneras evaluadas sobre todo en patas que tienen una baja heredabilidad, lo que permite una mayor permanencia en el hato con una vida productiva aceptable y reduciendo el porcentaje de descartes a edades tempranas.

Palabras claves: características lineales, profundidad corporal, heredabilidad, Holstein.

\section{Introducción}

En el Ecuador se producen 6,6 millones de leche cruda por día MAG, la región Sierra, se produce el 77,69\% de leche, en la Costa el 19,24\% y en la Amazonía 3,08\%. Cinco provincias con el mayor nivel de producción en el año 2019, las cuales conjuntamente generan el 61,70\% de la producción nacional de leche cruda: Pichincha (16,33\%), Manabí (12,34\%), Chimborazo (11,84\%), Cotopaxi (11,55\%), y Azuay (9,64\%). Según el ESPAC a relación de número de cabezas de ganado y su productividad en las diferentes regiones del país, se observa que la región con mayores rendimientos es la Sierra, con un promedio de 7,89 litros/vaca, seguido de la Amazonía con 4,50 litros/vaca, y finalmente se ubica la Costa con 4,31litros/vaca (Agrocalidad, 2014; Almeida, 2014). Esta diferencia respondería, entre otras cuestiones, al tipo de raza de ganado que se cría por región. La provincia de Chimborazo produce aproximadamente el 8\% del total de la producción de leche cruda de la Sierra. La población bovina en Chimborazo es 206.400, contando con 
vacas en producción en número de 59.900, con una producción de leche 405.360 litros (Álvarez, 2019).

La producción lechera beneficia a unos 279.000 ganaderos, los cuales se concentran principalmente en las provincias de Chimborazo (12\%), Manabí (11\%), Cotopaxi (11\%) y Pichincha (8\%) scpm.gob.ec. No menos de un millón y medio de personas viven directa e indirectamente de esta actividad (Álvarez, 2019; Ávila, 2006; Avilez et al., 2010). Entre los años 2013 a 2019, la producción registrada de litros de leche creció en 6,17\%; mientras entre 2016 y 2019 la producción nacional de leche se incrementó en un 25\%; con un promedio de crecimiento en los 10 últimos años de 18,5\%. Siendo importante el consumo de los ecuatorianos de 105 a 110 litros de leche per cápita por año HEIFER Y MAG, una cifra menor en comparación al consumo de lácteos en países como Uruguay y Argentina (Balarezo et al., 2016; Bencomo, 2010; Brown Swiss Association USA, 2015).

El uso y destino de la producción lechera en el país tiene un comportamiento regular, según las estimaciones del Ministerio de Agricultura y Ganadería, entre el 25\% y 32\% de la producción bruta se destina al consumo de terneros (autoconsumo) y mermas $2 \%$, la leche fluida disponible se destina en un $25 \%$ para la elaboración industrial (19\% leche pasteurizada y $6 \%$ para elaborados lácteos), el $75 \%$ entre consumo de utilización de la leche cruda (39\% para consumo humano directo y el $35 \%$ para industria caseras de quesos frescos, finalmente solamente el $1 \%$ se comercializa con Colombia en la frontera (Camargo, 2012; Cárdenas \& Guanga, 2018).

La especie bovina constituyen un grupo muy importante de animales, no sólo por su posibilidad de explotación y aporte en la economía de muchos países, sino por ser de las primeras especies domesticadas por el hombre (Cartuche et al., 2014; Contero et al., 2021). La ganadería es una actividad económica de origen muy antiguo que consiste en el manejo y explotación de animales domesticados por el hombre con fines de producción, para su aprovechamiento (Durán, 2012).

Según Echeverri \& Renault (2017), en bovinos existe una importante variabilidad genética, son animales de gran importancia económica y social dentro del sector agropecuario del país, capaces de producir grandes cantidades de leche, siendo la ganadería de leche una de las principales producciones en Ecuador, promoviendo beneficios a las pequeñas y grandes explotaciones, también es considerada una actividad familiar que aporta con el $45 \%$ de la producción nacional y aporta ingresos económicos 
en toda nuestra nación, hoy en día dicha actividad pecuaria es compleja y dispersa, sobre todo, por las situaciones que atraviesan actualmente los pequeños, medianos y grandes productores de leche (Estrella, 2015; Guapi et al., 2017).

Dentro de los ejes que priman a la producción animal tenemos: Sanidad Animal; Pastos y Forrajes; Nutrición y Alimentación; Reproducción y Mejoramiento Genético; Manejo; Sanidad e Industrialización en la cadena de suministro láctea, que son los pilares de todo proceso productivo y comercialización (Marini et al., 2021; Molina, 2005; Oscanoa, 2018). A ser la genética uno de los ejes importantes de la cadena del sistema de producción, ya que, según la variabilidad se analizan los diferentes genotipos para aplicar mejoramiento ganadero principalmente de los caracteres productivos, reproductivos, sanitarios y nutricionales para que, finalmente, la inversión en genética retorne el beneficio esperado (Peñafiel, 2016; Quinaluisa \& Benjamín, 2012). Es por ello que tomando como punto referencia estos problemas, con esta investigación se pretendió evaluar las vaquillas resultantes de las hembras del hato cruzadas con el o los sementales que se adaptaron mejor a las características y metas de la explotación, teniendo en cuenta que la aplicación de este plan de mejora fue con el fin de optimar la apariencia fenotípica de la progenie que va en paralelo con una eficiencia productiva (Quinaluisa \& Benjamín, 2012; Quinteros et al., 2017).

\section{Metodología}

En la presente investigación se utilizaron 12 vacas de raza Holstein mestizas en un rango de edad entre los 3 y 15 meses de edad de la Estación Experimental Tunshi, ubicada en la vía a Licto km 12, en el Cantón Riobamba, provincia de Chimborazo, a una altitud de $2735 \mathrm{msnm} ; 13^{\circ} \mathrm{C}$ temperatura; 558,60 $\mathrm{mm}$ de precipitación anual y $71 \%$ de humedad relativa.

La investigación tuvo una duración de 90 días, distribuidos en: selección de animales, evaluación de caracteres fenotípicos mejorados mediante la aplicación del SMS, registro genealógico. Para el análisis de datos se aplicó una estadística descriptiva, basada en el cálculo de porcentajes, media, mediana, moda y desviación estándar, las gráficas se realizaron a través de histogramas de frecuencias.

Las variables estudiadas se establecieron considerando los problemas de conformación de las vacas del hato, tomando en cuenta cada una de las características, presentes en los catálogos de pruebas de progenie. Todas ellas se midieron en valores absolutos, en una 
escala del 1 al 9 teniendo en cuenta los aspectos descritos anteriormente: Estatura, Fortaleza, Profundidad Corporal, Angulosidad (Carácter lechero), Ángulo de la Grupa, Anchura de la Grupa, Vista posterior de las patas, Vista lateral de las patas, Ángulo Podal.

Se realizó el análisis de las vaquillas resultantes del plan de mejoramiento usado previamente, posterior se seleccionó las hembras aptas para la evaluación; por medio de la información actualizada de las pruebas y pedigrí de dichos animales, se estableció las características fenotípicas mejoradas en la conformación de los animales. Se clasificó a las vaquillas de acuerdo a sus características físicas (variables), que pudieren estar aptas para el proceso. Para la evaluación de la aplicación del SMS, mediante la calificación lineal que hará el evaluador, se determinó el puntaje que alcanzaron las vaquillas, estableciendo así los posibles problemas que existen en el hato para posterior tomar decisiones y poder seguir realizando una mejora.

Mediante el manejo de registros se pudo establecer factores que ocasionen fallas en la selección, así controlar consanguinidad, haplotipos, etc., apoyándonos en el sistema a evaluar.

De acuerdo a los puntajes establecidos de los caracteres lineales, el evaluador observó las mayores debilidades en la conformación. Para finalmente en concordancia con las metas del hato, se escogió por medio del porcentaje las vaquillas aptas a futuro por cada característica fenotípica que procure mejorar la productividad del hato (Rodríguez, 2018).

\section{Marco teórico}

Warwick \& Legates (1980), manifiesta que el pedigrí es un registro de todos los animales que están emparentados con él. Si se da sólo la genealogía del individuo, el pedigrí es de valor muy limitado Desde el punto de vista de la práctica, el conocimiento de la productividad de los ancestros es necesario para que el pedigrí sea útil. Este tipo de información antes sólo existía para el ganado lechero, pero ahora existe también para el ganado de carne y para los cerdos.

Warwick \& Legates (1980), indica que se presta atención al pedigrí porque se carece de información adecuada sobre el mérito del individuo; en algunos casos, se necesita hacer algunas selecciones antes que el individuo exprese el carácter o los caracteres, como sería la ganancia de peso a partir de la alimentación. La información sobre el pedigrí es valiosa debido a que cada individuo recibe la mitad de sus genes a partir de cada uno de sus 
padres. Se debe recordar, sin embargo, que cada padre solo transmite la mitad de sus propios genes a sus descendientes.

\section{Calificación}

La Brown Swiss Association, USA (2015), manifiesta que la valoración del biotipo de los animales de granja es de gran importancia comercial y zootécnica, es practicado de forma sistemática por todas las asociaciones de raza pura del mundo, juzgar no se refiere a la condición corporal. La clasificación es la forma de medir qué tan cerca un animal llega a la ideal. Como todas las cosas, que han ido evolucionando con los años como: los objetivos de la raza y el medio ambiente en el que vive la vaca.

La Brown Swiss Association, USA (2015) argumenta que la información de clasificación tiene dos propósitos principales: la cría y comercialización. Como herramienta de cría, el criador puede mirar los puntajes lineales para ver donde su vaca necesita mejoras y seleccione apareamientos en consecuencia. Las puntuaciones lineales de cada hija entran en la información de resumen sire UDSA y describen las características de tipo de la descendencia de un toro. Todas las razas tienen un patrón de valoración que no es otra cosa que un listado de atributos que deben reunir los animales para poder ser evaluados y calificados.

\section{Calificación por tipo}

Almeida (2014), indica que la calificación por tipo es una estimación de la presunta utilidad de una vaca mediante la evaluación de su conformación.

\section{Calificación lineal}

Serrano \& Díaz (2009), indica que es necesario evaluar nuestro ganado nos permite realizar un buen proceso de selección con el fin de reproducir en nuestro hato sólo aquellos animales que presentan las mejores características. La clasificación lineal nos permite evaluar cada característica de forma independiente dándole un puntaje a cada ítem evaluado dependiendo de las desviaciones encontradas con respecto a lo que consideramos el estándar de cada raza.

Almeida (2014), indica que la clasificación lineal puede mejorar el tipo funcional de su rebaño y aumentar la producción. Cuando una vaca tiene tipo funcional correcto, ella tiene la habilidad de producir grandes volúmenes de leche por varias lactaciones. Como parte del programa de dicha clasificación, las vacas pueden ser evaluadas para 21 características funcionales o lineales.

La World Holstein Friesian Federation (2015), manifiesta que los rasgos descriptivos lineales son la base de los actuales sistemas de calificación del tipo y son el fundamento 
de todos los sistemas descriptivos de la vaca de leche. La calificación lineal está basada en las medidas de los caracteres del tipo individuales en vez de las opiniones. Esto describe el grado de los caracteres, no lo deseable que sea. Los valores lineales como herramienta de manejo son:

-Para uso en un programa de cruzamiento.

-Para administrar el progreso del mejoramiento del rebaño.

-Para comparar con sus contemporáneas de rebaño.

Serrano \& Díaz (2009), indica que los valores finales como herramientas de mercadeo son:

-Para darle un valor agregado universalmente aceptado al animal ante un comprador potencial.

-Para vender animales por pedigrí.

\section{Resultados}

\section{Estatura}

La estatura determinó una calificación media de 5,50 puntos $\pm 0,67$ puntos, es decir que los datos presentan una dispersión mínima, la mejor puntuación fue de 5 puntos representando un 58,34\% de las terneras evaluadas, y que corresponde a una estatura intermedia, seguida de la puntuación de 6 que representa un 33,33 \% del total de terneras y con una puntuación de 7 con un $8,33 \%$, Tabla 1 .

\section{Fortaleza}

Se aprecia una media general de 5,25 puntos $\pm 1,06$ puntos, en lo que respecta a las calificaciones individuales se aprecia que un 41,68\% de terneras alcanzan una calificación de 5 puntos; un 33,33 \% alcanzaron un puntaje de 6; y un 8,33\% de las terneras del hato lechero alcanzaron puntuaciones de 3,4 y 7 puntos, es necesario recordar que la fortaleza es la medida entre las dos patas delanteras en su parte más alta.

\section{Profundidad Corporal}

La profundidad corporal registró una media de 5,33 puntos \pm 1,56 puntos; que nos sirvió para comprobar que la muestra con una determinada media se considera como perteneciente a la población grupo de terneras. Al realizar la evaluación individual se aprecia que un 33,32 \% de las terneras, alcanzaron una puntuación de 7 puntos, mientras que $16,67 \%$ alcanzaron puntuaciones de 3, 4, 5 y 6 puntos en su orden, que equivale a un cuerpo poco profundo. 


\section{Tabla 1}

Caracteres lineales de terneras Holstein F1 mediante el programa de cruzamiento ganadero Select Mating Service (SMS). en el período 2014-2015 en la estación experimental Tunshi".

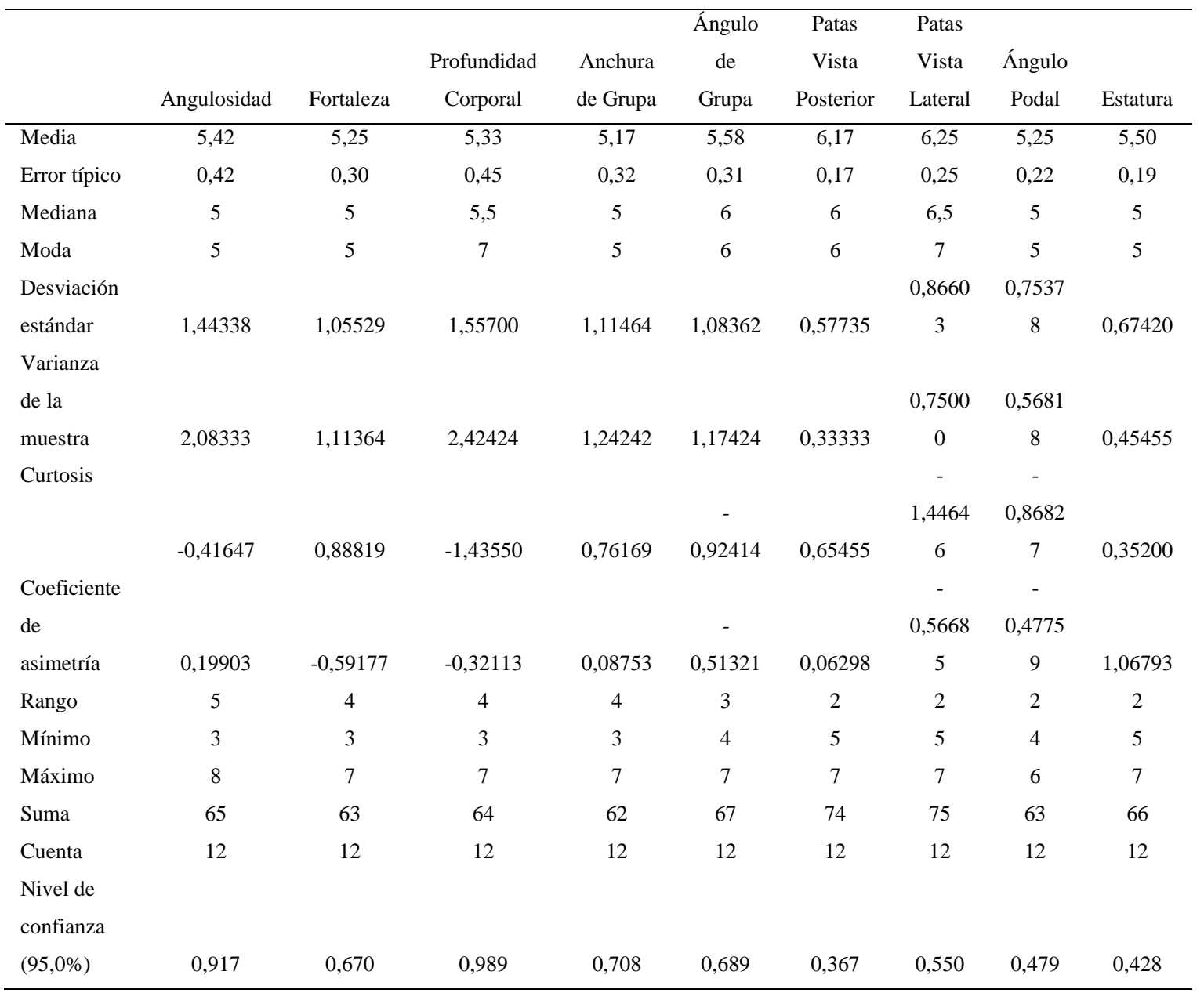

Fuente: Rodríguez (2018)

\section{Angulosidad (Carácter lechero)}

La angulosidad reportó una calificación media de 5,42 puntos $\pm 1,44$, el valor de 5 puntos es el más repetido, correspondiendo a un porcentaje promedio de $33,33 \%$, es decir vacas con un moderado ángulo de la costilla y separación, y una calidad de hueso ligeramente grosera y refinada respectivamente.

Ángulo de la Grupa

Registró un promedio de 5,58 puntos 1,08. La valoración de cada una de las vacas lecheras determinó que en un $50 \%$ de semovientes, registran una puntuación de 6 puntos es decir el íleon e isquion están ligeramente nivelados, continuando con la evaluación porcentual se aprecia que en un $25 \%$ se identifica con una calificación de 4 puntos. 


\section{Anchura de la Grupa}

Se determinó una media general de 5,17 puntos $\pm 1,11$ puntos y que corresponde a un ancho de anca amplio, se reportó que en el 58,33 \% del total de terneras se aprecia una calificación de 5 puntos, es decir vacas con un ancho de anca aceptable o promedio, seguida de las calificaciones de 7 puntos alcanzados por el 16,68\% con una achura de anca intermedia; así como también en un $8,33 \%$ de terneras equitativamente que registraron una calificación de 6, 4, 3 puntos.

\section{Vista posterior de las patas}

Se estableció una media general de 6,17 puntos \pm 0,58 puntos. La valoración porcentual de la calificación de las patas vistas por atrás de las terneras del hato de la Estación Experimental Tunshi, reportó que en el 66,67 \% del total se aprecia una calificación de 6 puntos, es decir pezuñas ligeramente abiertas, corvejón ligeramente cerrado, seguida de las calificaciones de 7 puntos que corresponde el $25 \%$; mientras tanto que en el 8,33\% se determinó una calificación de 5, es decir terneras con pezuñas poco abiertas, corvejón poco cerrado.

\section{Vista lateral de las patas}

Para la presente variable se determinó una media de 6,25 puntos $\pm 0,87$ puntos por lo que se registró que un 50\% alcanzaron una puntuación de 7 puntos, es decir presentan pezuñas ligeramente abiertas, corvejón ligeramente cerrado, seguida por la calificación de 6 y 5 puntos que fue un $25 \%$ cada puntaje del grupo de animales.

\section{Ángulo podal}

El ángulo de las pezuñas de las terneras del hato lechero de la Estación Experimental Tunshi, registró una media general de 5,25 puntos $\pm 0,75$ puntos que es el valor de la desviación estándar.

\section{Discusión}

Según la Asociación Holstein Friesian USA (2015), Es una de las dos razas lecheras más pesadas. Su característica más importante es el tamaño, asociado con el grado adecuado de refinamiento lechero, a efectos de disponer de un animal que produzca cantidades elevadas de leche en forma sostenida.

Las terneras extremadamente grandes, para nuestras condiciones de manejo son ineficientes, una característica importante de la raza Holstein es su tamaño asociado con un grado adecuado de refinamiento lechero, se pone hincapié en el tamaño a efectos de disponer de un animal que produzca cantidades elevadas de leche en forma sostenida. 
Para nuestro clima, se busca una vaca mediana, que tenga una puntuación de 5 y 6 en promedio como escala de referencia, que son más eficientes y eficaces en la transformación de pasto en leche.

Según Zhicay (2016), la variable fortaleza de la vaca lechera mide la anchura del pecho, la substancia del hueso de la parte delantera de la vaca. En lo que respecta a los rasgos descriptivos lineales son la base de los actuales sistemas de calificación del tipo y son el fundamento de todos los sistemas descriptivos de la vaca de leche. La calificación lineal está basada en las medidas de los caracteres del tipo individuales en vez de las opiniones. La profundidad corporal es la distancia encontrada entre el dorso de la vaca y la parte más baja del barril. Se mide al nivel de la última costilla y su punto de referencia es óptico. Lo mencionada Serrano \& Díaz (2009), la estructura ósea y muscular es más importante en toros que en vacas, sin embargo, las vacas también deben tener una buena conformación con buena angularidad, teniendo en cuenta de no utilizar toros con demasiada angularidad en vacas refinadas ya que tendremos crías muy frágiles, algo no muy deseado para nuestras condiciones de manejo.

Esta característica se define mediante la colocación de los isquiones en las grupas. Es una medida que va desde el isquion al ilion y el ángulo que comprende entre estas. El ángulo de anca está relacionado con el comportamiento reproductivo de la futura vaca, puesto que la colocación del isquion es la que determina, permite o limita el drenaje apropiado del canal del parto. Si existen desproporciones de la punta del ilion al isquion habrá problemas en el parto (Terán, 2019; Vargas et al., 2011).

\section{Conclusiones}

- Al evaluar las terneras hijas de las vacas que están en el programa de cruzamiento SMS se observó una mejora en las características lineales sobre todo en patas que tiene una baja heredabilidad, esto permitirá una mayor permanencia en el hato con una vida productiva aceptable y reduciendo el porcentaje de descartes a tempranas edades.

- La mayoría de las características lineales evaluadas en las terneras Holstein Fresian Mestizas de la estación Experimental Tunshi, presentaron calificaciones promedio comparadas con el ideal de la raza.

- Debido a que las madres de estas terneras evaluadas tenían problemas severos en patas y ubres delanteras, al momento de evaluar y ranquear se observó que se ha 
mejorado notablemente la característica de componentes en patas, cabe recalcar que para fijar esta característica se necesita 3 generaciones pero con los toros utilizados nos ayudó a ganar tiempo en la selección y mejoramiento del hato de Tunshi, ya que en la primera generación se vio cambios por encima de las expectativas, la característica de ubre delantera no se evaluó por razón de que son animales en crecimiento y no en producción.

- Seguir utilizando toros por inseminación artificial que trasmitan altos valores en las características en las cuales el hato de Tunshi tiene deficiencias y así tener una selectividad a menor tiempo y llegar a tener animales con una conformación aceptable.

- Procurar crear antecedentes genealógicos y registros significativos de todos los animales de la estación experimental Tunshi, para determinar las características lineales más representativas de los animales, el promedio genético para producción, longevidad, salud y conformación, identificación de animales superiores corregir errores de consanguinidad y aparecimiento de problemas sobre todo de patas.

- Realizar una selección rigurosa de las terneras para que los problemas no persistan y en el futuro tener animales que no tengan dificultades y ser descartados en sus primeros partos. Aplicar las recomendaciones del evaluador en cuanto a selección de animales y en lo que respecta a la inversión en la compra de semen de toros genéticamente superiores, de acuerdo a las características que se quieren mejorar, esto permitirá el aumento de la vida productiva del animal.

- Realizar una tesis con estos animales cuando estén en producción para observar las características de conformación de ubre y poder avaluar los toros recomendados por el programa SMS y su porcentaje de mejora en valores y tiempo

\section{Referencia Bibliográficas}

Agrocalidad. (2014). Programa de Vacunación Fiebre Aftosa (segunda fase). Riobamba: AGROCALIDAD. Recuperado el 26 de mayo de 2014, de 2013.

Almeida, F. (2014). Manual de juzgamiento ganadero. Riobamba-Ecuador: ESPOCH. pp. 12 - 56.

Álvarez, Hualpa KA. (2019). Importancia mineral en vacas lecheras en Ecuador. 2019. 
Ávila B, Luis G. (2006). Técnicas bovinométricas para determinar la capacidad reproductiva, productiva de hembras lecheras de la raza Holstein de la región austral. 2006.

Avilez JP, Escobar P, Von Fabeck G, Villagran K, García F, Matamoros R, et al. (2010). Caracterización productiva de explotaciones lecheras empleando metodología de análisis multivariado. Revista Científica. 2010;20(1):74-80.

Balarezo LR, García-Díaz JR, Hernández-Barreto MA, García López R. (2016). Estado metabólico y reproductivo del ganado Holstein en la región del Carchi, Ecuador. Cuban Journal of Agricultural Science. 2016;50(3):381-92.

Bencomo BG. (2010).Manejo sanitario eficiente del ganado bovino: Principales enfermedades. Instituto Nicaragüense de Tecnología Agropecuaria, Managua (Nicaragua).

Brown Swiss Association USA. (2015). Programa de clasificación por tipo en razas de doble propósito. Recuperado el 15 de Julio 2016, obtenido de sitio web de BrownSwissAssosation:http://www.brownswissusa.com/Programs/Classification /tabid/136/Default.aspx

Camargo, O. (2012). La vaca lechera: Entre la eficiencia económica y la ineficiencia biológica. Archivos de Zootecnia. 2012;61(237):13-29.

Cárdenas MAR, Guanga LEA. (2018). Caracterización morfométrica e índices zoométricos de los grupos raciales bovinos existentes en los cantones occidentales de la provincia del Azuay. Centrosur. 2018;1(1):43-51.

Cartuche L, Vargas N, Pascual M, editors. (2014). Análisis preliminar del pedigrí de las razas bovinas lecheras Jersey y Brown Swiss en el Ecuador 2014.

Contero R, Requelme N, Cachipuendo C, Acurio D. (2021). Calidad de la leche cruda y sistema de pago por calidad en el Ecuador. LA GRANJA Revista de Ciencias de la Vida. 2021;33(1):31-43.

Durán Torres, JP. (2012). Análisis de correlación y regresión entre los caracteres fenotípicos del tipo lechero, con la producción lechera alcanzada de Vacas Holstein Friesian, en la cuenca lechera de Machachi. 2012.

Echeverri Pinilla A, Renault Adib A. (2017). Guía para la generación participativa de políticas públicas diferenciadas para la agricultura familiar. 2017.

Estrella Casanova, FP. (2015). Evaluación del hato lechero de la Estación Experimental Tunshi, utilizando el programa de cruzamiento ganadero select mating service (SMS). 2015.

Guapi Guamán RA, Masaquiza Moposita D, Curbelo Rodríguez LM. (2017). Caracterización de sistemas productivos lecheros en condiciones de montaña, Parroquia Químiag, Provincia Chimborazo, Ecuador. Revista de Producción Animal. 2017;29(2):14-24. 
Marini PR, Biga P, Di-Masso RJ. (2021). Caracterización multivariada de la eficiencia productivo-reproductiva y edad al primer parto en vacas Holstein. Agronomía Mesoamericana. 2021;32(1):34-44.

Molina, S. (2005). Evaluación agronómica y bromatológica del pasto Maralfalfa (Pennisetum sp.) cultivado en el Valle del Sinú. Rev Fac Nac Agron Colombia. 2005;58(1):39.

Oscanoa Montes, NR. (2018). La genética en la reproducción de los animales. 2018.

Peñafiel Siguencia, RD. (2016). Evaluación de hato lechero del Centro de Excelencia Agropecuario de Burgay, utilizando el programa de cruzamiento ganadero Select Mating Service (SMS). 2016.

Quinaluisa A, Benjamín F. (2012). Valoración Biotipológica y Caracterización Zoométrica del Grupo Genético Bovino Autóctono Pizán. 2012.

Quinteros Pozo OR, Vargas Burgos JC, Barbona I, Marini PR. (2017). Indicadores metabólicos sanguíneos de genotipos lecheros en pastoreo de la provincia de Napo-Ecuador. LA GRANJA Revista de Ciencias de la Vida. 2017;26(2):119-30.

Requelme N, Bonifaz N. (2012). Caracterización de sistemas de producción lechera de Ecuador. La Granja. 2012;15(1):55-69.

Rodríguez Paspuel DM. (2018).Valoración fenotípica de terneras obtenidas mediante el programa de cruzamiento gandero Select Mating Service (SMS) en el período 2014-2015 en la Estación Experimental Tunshi.

Serrano Estrada A, Díaz García F. (2009). Relación de medidas bovinométricas y medidas lineales de la ubre con producción de leche y con la edad al primer parto en vacas primerizas Holstein en el CIC Santamaría del Puyón de la Universidad de La Salle. 2009.

Terán Flores, JM. (2019). Análisis del mercado de la leche en Ecuador: factores determinantes y desafíos. 2019.

Vargas J, Benítez D, Torres V, Velázquez F, Erazo O. (2011). Tipificación de las fincas ganaderas en el piedemonte de las provincias Los Ríos y Cotopaxi de la República del Ecuador. Revista Cubana de Ciencia Agrícola. 2011;45(4):381-90.

Warwick, E, Legates, J. (1980). Cría y Mejora del ganado (3ª ed) México: Mcgraw-Hill pp. 23 - 59.

World Holstein Friesian Federation. (2015). Rasgos representativos en la raza Holstein Recuperado en Enero de 2016, de Sitio web de World Holstein Friesian Federation. http://www.whff.info/info/typetraits/type_esp_2005-2.pdf.

Zhicay Orellana, WO. (2016). Valoración de rasgos morfométricos y productivos de vacas holstein mestizo y puras en el cantón Chambo. 


\section{PARA CITAR EL ARTÍCULO INDEXADO}

Rodríguez Paspuel, D., Almeida López, F., \& Toalombo Vargas, P. (2021). Evaluación de caracteres lineales de terneras Holstein F1 mediante el programa de cruzamiento ganadero Select Mating Service (SMS). AlfaPublicaciones, 3(3.2), 112-125. https://doi.org/10.33262/ap.v3i3.2.120

\section{Ciencia Digital \\ Editorial}

El artículo que se publica es de exclusiva responsabilidad de los autores y no necesariamente reflejan el pensamiento de la Revista Alfa Publicaciones.

El artículo queda en propiedad de la revista y, por tanto, su publicación parcial y/o total en otro medio tiene que ser autorizado por el director de la Revista Alfa Publicaciones.
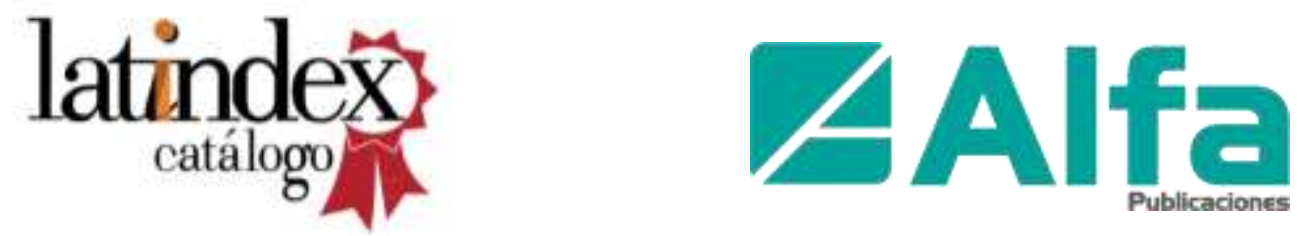